\title{
Qubit decoherence by low-frequency noise
}

\author{
K. Rabenstein, V.A. Sverdlov, and D.V. Averin \\ Department of Physics and Astronomy, Stony Brook University, SUNY, Stony Brook, NY 11794-3800
}

(Dated: June 16, 2018)

\begin{abstract}
We have derived explicit non-perturbative expression for decoherence of quantum oscillations in a qubit by low-frequency noise. Decoherence strength is controlled by the noise spectral density at zero frequency while the noise correlation time $\tau$ determines the time $t$ of crossover from the $1 / \sqrt{t}$ to the exponential suppression of coherence. We also performed Monte Carlo simulations of qubit dynamics with noise which agree with the analytical results and show that most of the conclusions are valid for both Gaussian and non-Gaussian noise.
\end{abstract}

PACS numbers: 03.65.Yz, 03.67.Lx, 72.70.+m

Despite the large number of successful demonstrations of coherent quantum oscillations in individual 1, 2, 3, [4, 5, 6, 7, 8] and coupled [9] Josephson-junction qubits, quantitative understanding of these oscillations is so far limited. The main area of discrepancy between experiment and theory is qubit decoherence. The typical quality factors of reported oscillations, while not as large as required by potential applications in quantum computation, are still quite large in physics term (typically not less than $20 \div 30$ ). This fact should imply weak decoherence describable by the standard perturbation theory in qubit-environment coupling (see, e.g., [10]). Several basic features of this theory, however, do not agree with experimental observations. Most importantly, observed decay time $T_{2}$ of coherent oscillations is typically shorter than the energy relaxation time $T_{1}$ even at optimal qubit bias points [3, 4, 11] where perturbation theory predicts no pure dephasing terms. Another discrepancy is between the observed two-qubit decoherence rate 9$]$ and its values that can be obtained from the perturbation theory under natural assumptions 12 .

Qualitatively, the basic reason for discrepancy between $T_{1}$ and $T_{2}$ is the low-frequency noise that can reduce $T_{2}$ without changing significantly the relaxation rates. Mechanisms of low-frequency, or specifically $1 / f$, noise exist in all solid-state qubits: background charge fluctuations for charge-based qubits 13., impurity spins or trapped fluxes for magnetic qubits [14]. Manifestations of this noise are observed in the echo-type experiments 11. Low-frequency noise for qubits is also created by the electromagnetic fluctuations in filtered control lines.

The goal of our work is to develop quantitative theory of low-frequency decoherence by studying qubit dynamics under the influence of noise with small characteristic amplitude $v_{0}$ and long correlation time $\tau$. In the case of Gaussian noise, we obtained explicit non-perturbative expression describing decay in time of coherent qubit oscillations. The strength of decoherence in this expression is controlled by the noise spectral density at zero frequency, $v_{0}^{2} \tau$. For long correlation times $\tau \gg \Delta^{-1}$, where $\Delta$ is the qubit tunnel amplitude, the spectral density $v_{0}^{2} \tau$ can be large even for weak noise $v_{0} \ll \Delta$ and our analyt- ical results are exact as function of $v_{0}^{2} \tau$ in this limit. We also performed direct numerical simulations of the lowfrequency qubit decoherence. The simulation results confirm analytical expressions for Gaussian noise and show that our main conclusions: cross-over from the $1 / \sqrt{t}$ to the exponential suppression of coherence at time $t \simeq \tau$; and the strength of decoherence controlled by the noise spectral density $S_{v}(0)$ at zero frequency remain valid for non-Gaussian noise. The decoherence rates for Gaussian and non-Gaussian noise behave, however, quite differently as functions of $S_{v}(0)$. In physics terms, the two noise models we study correspond, respectively, to lowfrequency electromagnetic noise, and noise of localized excitations in the situation when only a few excitations with comparable time scales are coupled to the qubit.

The Hamiltonian of a qubit with a fluctuating bias energy $v(t)$ (see inset in Fig. 1b) is:

$$
H=-\frac{1}{2}\left[\Delta \sigma_{x}+(\varepsilon+v(t)) \sigma_{z}\right],
$$

where $\varepsilon$ is the average bias, and $\sigma$ 's here and below denote Pauli matrices. In this work, we mostly focus on the situation when the noise $v(t)$ has characteristic correlation time $\tau$, i.e., the noise correlation function and its spectral density can be taken as

$$
\left\langle v(t) v\left(t^{\prime}\right)\right\rangle=v_{0}^{2} e^{-\left|t-t^{\prime}\right| / \tau}, \quad S_{v}(\omega)=\frac{2 v_{0}^{2} \tau}{1+(\omega \tau)^{2}},
$$

where $v_{0}$ is the typical noise amplitude and $\langle\ldots\rangle$ denotes average over different realizations of noise. We assume that the temperature $T$ of the noise-producing environment is large on the scale of the cut-off frequency $1 / \tau$, and it can be treated as classical. (In the regime of interest, $1 / \tau \ll \Delta$, the temperature can obviously be still small on the qubit energy scale.)

The two effects of the weak noise on the dynamics of the qubit (1) are the transitions between two energy eigenstates with energies $\pm \Omega / 2, \Omega \equiv\left(\Delta^{2}+\varepsilon^{2}\right)^{1 / 2}$, and "pure" (unrelated to transitions) dephasing that suppresses coherence between these states. Within the standard perturbation theory, the transition rate is proportional to $S_{v}(\Omega)=v_{0}^{2} / \Omega^{2} \tau$. One can see that the condition of weak noise $v_{0} \ll \Delta$ makes the transition rate small 
compared both to $\Delta$ and $1 / \tau$ ensuring that the perturbation theory is sufficient for the description of transitions. As discussed qualitatively in the introduction, the fact that the noise correlation time is long, $\tau \gg \Delta^{-1}$, makes the perturbation theory inadequate for the description of pure dephasing. For low-frequency noise, a proper (nonperturbative in $v_{0}^{2} \tau$ ) description is obtained by looking at the accumulation of the noise-induced phase between the two instantaneous energy eigenstates. If $v_{0} \ll \Delta$, one can determine the rate of accumulation of this phase by expanding the energies in noise amplitude $v(t)$. Also, in this case the dephasing rate is larger than the transition rate and can be calculated disregarding the transitions. The factor $F(t)$ describing suppression in time of coherence between the two states (i.e., suppression of the offdiagonal element $\rho_{12}$ of the qubit density matrix in the energy basis: $\left.\rho_{12}(t)=F(t) \rho_{12}(0) e^{-i \Omega t}\right)$ can be written then as follows:

$$
F(t)=\left\langle\exp \left\{-i \int_{0}^{t}\left[\frac{\varepsilon v\left(t^{\prime}\right)}{\Omega}+\frac{\Delta^{2} v^{2}\left(t^{\prime}\right)}{2 \Omega^{3}}\right] d t^{\prime}\right\}\right\rangle .
$$

For Gaussian noise, the correlation function (2) determines the noise statistics completely. In this case, it is convenient to take the average in Eq. (3) by writing it as a functional integral over noise. For this purpose, and also for use in the numerical simulations, we start with the "transition" probability $p\left(v_{1}, v_{2}, \delta t\right)$ for the noise to have the value $v_{2}$ a time $\delta t$ after it had the value $v_{1}$ :

$$
\begin{array}{r}
p\left(v_{1}, v_{2}, t\right)=\left[2 \pi v_{0}^{2}\left(1-e^{-2 \delta t / \tau}\right)\right]^{-1 / 2} \times \\
\exp \left\{-\frac{1}{2 v_{0}^{2}} \frac{\left(v_{2}-v_{1} e^{-\delta t / \tau}\right)^{2}}{1-e^{-2 \delta t / \tau}}\right\} .
\end{array}
$$

Using this expression we introduce the probability of specific noise realization as $p_{0}\left(v_{1}\right) \cdot p\left(v_{1}, v_{2}, \delta t_{1}\right) \cdot p\left(v_{2}, v_{3}, \delta t_{2}\right)$. $\ldots$, where $p_{0}(v)=\left(2 \pi v_{0}^{2}\right)^{-1 / 2} \exp \left\{-v^{2} / 2 v_{0}^{2}\right\}$ is the stationary Gaussian probability distribution of $v$. Taking the limit $\delta t_{j} \rightarrow 0$ we see that the average over the noise can be written as the following function integral:

$$
\begin{array}{r}
\langle\ldots\rangle=\int d v(0) d v(t) D v\left(t^{\prime}\right) \ldots \\
\times \exp \left\{-\frac{v(0)^{2}+v(t)^{2}}{4 v_{0}^{2}}-\frac{1}{4 v_{0}^{2} \tau} \int_{0}^{t} d t^{\prime}\left(\tau^{2} \dot{v}^{2}+v^{2}\right)\right\} .
\end{array}
$$

Since the average in Eq. (3) with the weight (15) is now given by the Gaussian integral, it can be calculated straightforwardly:

$$
\begin{aligned}
& F(t)=F_{0}(t) \exp \left[-\alpha^{2}\left(\frac{\nu t}{\tau}-2\left[\operatorname{coth} \frac{\nu t}{2 \tau}+\nu\right]^{-1}\right)\right], \\
& F_{0}(t)=e^{t / 2 \tau}\left[\cosh (\nu t / \tau)+\frac{1+\nu^{2}}{2 \nu} \sinh (\nu t / \tau)\right]^{-1 / 2},
\end{aligned}
$$

where $\nu \equiv \sqrt{1+2 i v_{0}^{2} \Delta^{2} \tau / \Omega^{3}}$ and $\alpha \equiv \varepsilon \tau v_{0} / \Omega \nu^{3 / 2}$.

Equation ([6) is our main analytical result for dephasing by the Gaussian noise. To analyze its implications, we
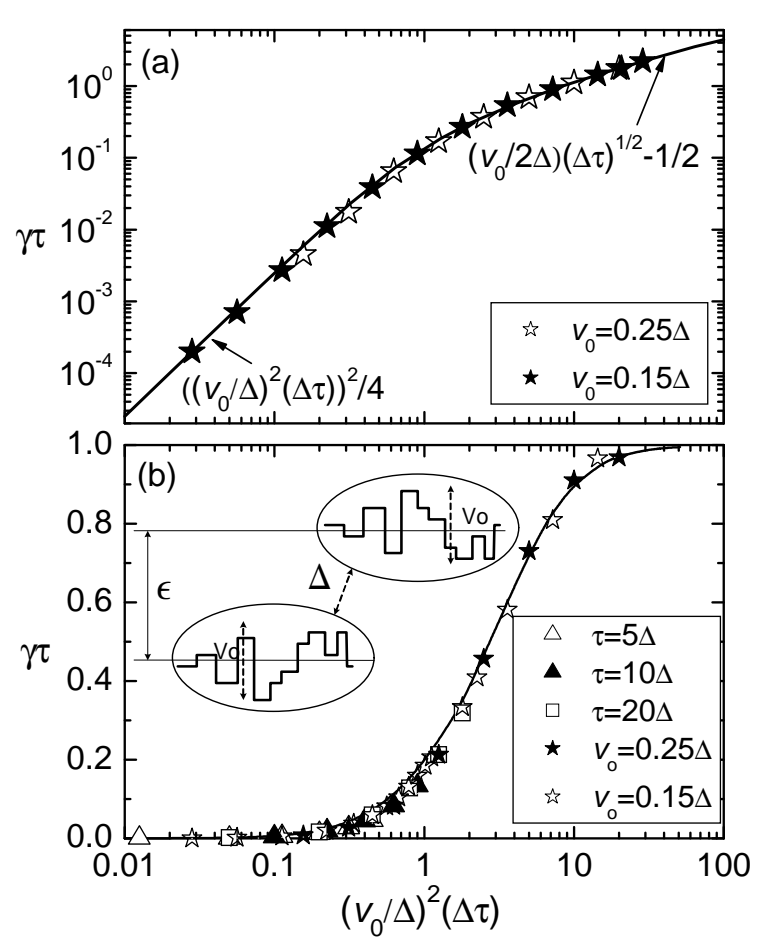

FIG. 1: The rate $\gamma$ of exponential qubit decoherence at long times $t \gg \tau$ for $\varepsilon=0$ and (a) Gaussian and (b) a model of the non-Gaussian noise with characteristic amplitude $v_{0}$ and correlation time $\tau$. Solid lines give analytical results: Eq. (8) in (a) and Eq. (13) in (b). Symbols show $\gamma$ extracted from Monte Carlo simulations of qubit dynamics. Note different scales for $\gamma$ in parts (a) and (b). Inset in (b) shows schematic diagram of qubit basis states fluctuating under the influence of noise $v(t)$.

start with the case $\varepsilon=0$, where pure qubit dephasing vanishes in the standard perturbation theory. Dephasing (6) is still non-vanishing and its strength depends on the noise spectral density at zero frequency $S_{v}(0)=2 v_{0}^{2} \tau$ through $\nu=\sqrt{1+i s}, s \equiv S_{v}(0) / \Delta$. For small and large times $t$ Eq. (6) simplifies to:

$$
F(t)= \begin{cases}{\left[\frac{1+t / \tau}{1+t / \tau+i s t / 2 \tau}\right]^{1 / 2},} & t \ll \tau, \\ 2 \sqrt{\nu} e^{-(\gamma+i \delta) t} /(1+\nu), & t \gg \tau,\end{cases}
$$

where

$$
\gamma=\frac{1}{2 \tau}\left[\left(\frac{\left(1+s^{2}\right)^{1 / 2}+1}{2}\right)^{1 / 2}-1\right] .
$$

Besides suppressing coherence, the noise also shifts the frequency of qubit oscillations. The corresponding frequency renormalization is well defined for $t \gg \tau$ :

$$
\delta=\frac{1}{2 \tau}\left[\frac{\left(1+s^{2}\right)^{1 / 2}-1}{2}\right]^{1 / 2} .
$$


Suppression of coherence (77) for $t \ll \tau$ can be qualitatively understood as the result of averaging over the static distribution of noise $v$. In contrast to this, at large times $t \gg \tau$, the noise appears to be $\delta$-correlated, the fact that naturally leads to the exponential decay (7). This interpretation means that the two regimes of decay should be generic to different models of the low-frequency noise. Indeed, they exist for the non-Gaussian noise considered below, and are also found for Gaussian noise with $1 / f$ spectrum [15]. Crossover between the two regimes takes place at $t \simeq \tau$, and the absolute value of $F(t)$ in the crossover region can be estimated as $\left(1+s^{2}\right)^{-1 / 4}$, i.e. $s$ determines the amount of coherence left to decay exponentially. The rate (8) of exponential decay shows a transition from the quadtratic to square-root behavior as a function of $S_{v}(0)$ that can be seen in Fig. 1a, which also shows the decay rate extracted from numerical simulations of Gaussian noise. (Numerical procedure is discussed below.) One can see that our analytical and numerical results agree well for quite large noise amplitude $v_{0}$.

Non-zero qubit bias $\varepsilon$ leads to additional dephasing $F(t) / F_{0}(t)$ described by the last exponential factor in Eq. (6). One can see that similar to $F_{0}(t)$ additional dephasing exhibits the crossover at $t \simeq \tau$ from "inhomogenious broadening" (averaging over the static distribution of the noise $v$ ) to exponential decay at $t \gg \tau$. In contrast to $F_{0}(t)$, the short-time decay is now Gaussian:

$$
\ln \left[\frac{F(t)}{F_{0}(t)}\right]=-\frac{\varepsilon^{2}}{\Omega^{2}} \cdot\left\{\begin{array}{cc}
v_{0}^{2} t^{2} / 2, & t \ll \tau, \\
v_{0}^{2} \tau t /\left(1+i s(\Delta / \Omega)^{3}\right), & t \gg \tau .
\end{array}\right.
$$

We see that, again, the rate of exponential decay depends non-trivially on the noise spectral density $S_{v}(0)$, changing from direct to inverse proportionality to $S_{v}(0)$ at small and large $s$, respectively.

Our approach can be used to calculate the rate of exponential decay at large times $t$ for Gaussian noise with arbitrary spectral density $S_{v}(\omega)$. Such a noise can be represented as a sum of noises (2) and appropriate transformation of variables in this sum enables one to write the average over the noise as a functional integral similar to (5). For calculation of the relaxation rate at large $t$, the boundary terms in the integral (5) can be neglected and it is dominated by the contribution from the "bulk" which can be conveniently written in terms of the Fourier components

$$
v_{n}=(2 / t)^{1 / 2} \int_{0}^{t} d t^{\prime} v\left(t^{\prime}\right) \sin \omega_{n} t^{\prime}, \quad \omega_{n}=\pi n / t .
$$

Then, $\langle\ldots\rangle=\int D v \ldots \exp \left\{-(1 / 2) \sum_{n}\left|v_{n}\right|^{2} / S_{v}\left(\omega_{n}\right)\right\}$. Combining this equation and Eq. (3) we get at large $t$ :

$$
\begin{aligned}
& F(t)=\exp \left\{-\frac{t}{2}\left[\frac{\varepsilon^{2} \Omega S_{v}(0)}{\Omega^{3}+i S_{v}(0) \Delta^{2}}+\right.\right. \\
& \left.\left.\frac{1}{\pi} \int_{0}^{\infty} d \omega \ln \left(1+i S_{v}(\omega) \Delta^{2} / \Omega^{3}\right)\right]\right\} .
\end{aligned}
$$

For unbiased qubit, $\varepsilon=0$, this equation coincides with the one obtained by more involved diagrammatic perturbation theory in quadratic coupling [15].

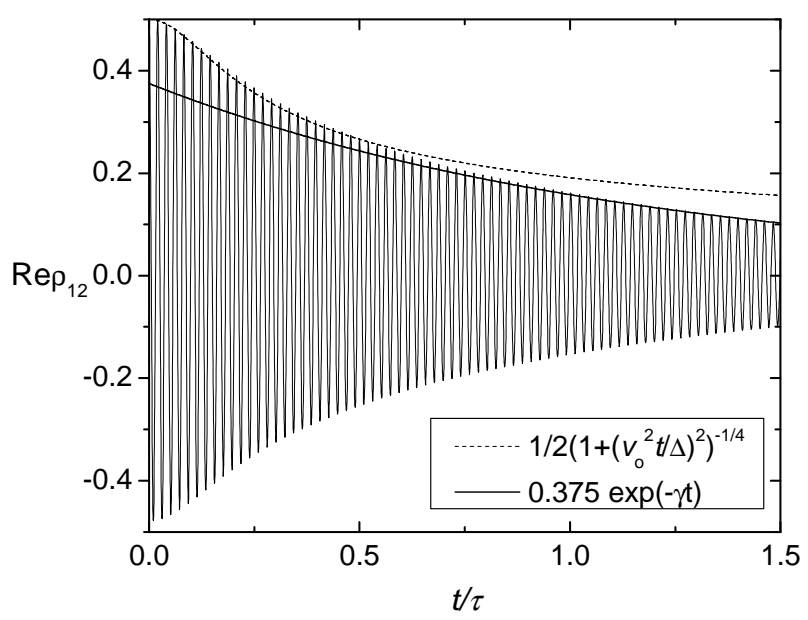

FIG. 2: The profile of coherent quantum oscillations in an unbiased qubit dephased by the non-Gaussian noise with characteristic amplitude $v_{0}=0.15 \Delta$ and correlation time $\tau=300 \Delta^{-1}$ obtained by direct simulation of qubit dynamics with noise. Solid line is the exponential fit of the oscillation amplitude at large times. Dashed line is the initial $1 / \sqrt{t}$ decay caused by effectively static distribution of $v$.

To check how well the analytic theory described above works for finite noise amplitude $v_{0}$, and to see how sensitive the results are to the assumption of the Gaussian noise, we performed Monte Carlo simulations of the qubit oscillations under the influence of Gaussian and nonGaussian noise. We looked specifically at the coherent oscillations of a qubit with Hamiltonian (11) that start in one of the eigenstates of the $\sigma_{z}$ operator, focusing on the case $\varepsilon=0$. The qubit density matrix was averaged over up to $10^{7}$ realizations of noise. In the case of Gaussian noise, realizations were built using the transition probability (4). For non-Gaussian noise we used the model of Ref. 16, which should provide an appropriate description of the situation when a qubit is coupled to several fluctuators with similar characteristic time scale $\tau$ of the fluctuations. In this model the fluctuators create random qubit bias $v$ which remains constant for some (random) time interval after which it is updated and the new value remains constant during the next time interval, etc. The time intervals between bias updates are taken to be distributed according to the Poisson distribution with characteristic time $\tau$. For more direct comparison with the Gaussian noise, we assumed Gaussian distribution $p_{0}(v)$ of $v$. The correlation function of $v(t)$ defined in this way is given by the same Eq. (2).

Example of the oscillations dephased by such a noise is given in Fig. 2. It shows real part of the off-diagonal 
element $\rho_{12}(t)$ of the qubit density matrix in the energy eigenstates basis. For oscillations starting in one of the $\sigma_{z}$ eigenstates, $\rho_{12}(0)=1 / 2$. Similarly to the case of Gaussian noise, we consider only weak noise, $v_{0} \ll \Delta$. In this case, there is a crossover at $t \simeq \tau$ in the oscillation amplitude from the initial $1 / \sqrt{t}$ suppression of coherence due to averaging over static potential distribution: $\rho_{12}(t)=\rho_{12}(0) /\left(1+i v_{0}^{2} t / \Delta\right)^{1 / 2}$ (neglecting all terms of order $t / \tau)$, to exponential suppression at $t \gg \tau$.

The rate of the exponential decay can be found analytically as follows. Expansion of the average qubit density matrix $\rho(t)$ in the number of "jumps" of $v(t)$ leads to the Dyson-like equation for its evolution [16]:

$$
\begin{array}{r}
\rho(t)=e^{-t / \tau}\left\langle S(t, 0) \rho(0) S^{\dagger}(t, 0)\right\rangle \\
+\int_{0}^{t} \frac{d t^{\prime}}{\tau} e^{-\left(t-t^{\prime}\right) / \tau}\left\langle S\left(t, t^{\prime}\right) \rho\left(t^{\prime}\right) S^{\dagger}\left(t, t^{\prime}\right)\right\rangle,
\end{array}
$$

where $\langle\ldots\rangle$ denotes the average over the distribution of $v$. For weak noise, introducing slowly-varying amplitude $r$ of $\rho_{12}(t)=r(t) e^{-i \Delta t}$, one can reduce Eq. (11) to the equation for $r(t)$ neglecting rapidly oscillating terms:

$$
\begin{array}{r}
r(t)=e^{-t / \tau} r(0)\left(1+i v_{0}^{2} t / \Delta\right)^{-1 / 2} \\
+\int_{0}^{t} \frac{d t^{\prime}}{\tau} e^{-\left(t-t^{\prime}\right) / \tau} r\left(t^{\prime}\right)\left(1+i v_{0}^{2}\left(t-t^{\prime}\right) / \Delta\right)^{-1 / 2}
\end{array}
$$

With the exponential ansatz for $r(t): r(t) \propto e^{-(1-\lambda) t / \tau}$ Eq. (12) gives then equation for the parameter $\lambda$ :

$$
\lambda=\int_{0}^{\infty} d x e^{-x}\left[1+\frac{i x s}{2 \lambda}\right]^{-1 / 2} .
$$

(Omission of the first term in Eq. (12) is justified by the final result for the oscillation decay rate $\gamma=(1-$ $\operatorname{Re}[\lambda]) / \tau$.) Asymptotics of $\gamma$ found from Eq. (13) are:

$$
\gamma=\frac{1}{\tau} \times\left\{\begin{array}{cl}
s^{2} / 8, & s \ll 1, \\
1-16 \pi / s^{2}, & s \gg 1 .
\end{array}\right.
$$

The rate $\gamma(s)$ evaluated from Eq. (13) is shown in Fig. $1 \mathrm{~b}$, together with the pure dephasing rates found numerically by fitting the oscillation amplitude (similar to that shown in Fig. 2) at $t \gg \tau$ and subtracting the contribution $S_{v}(\Delta)$ to dephasing from real transitions. One can see that Eq. (13) indeed gives an accurate description of pure dephasing rates. Similarly to the case of Gaussian noise, $\gamma$ depends only on $S_{v}(0)$. In both situations, $\gamma \propto v_{0}^{4} \tau / \Delta^{2}$ for small $v_{0}^{2} \tau / \Delta$, the fact that can be explained by the lowest-order perturbation theory in qubit energy fluctuations. In the non-perturbative regime, however, the behavior of $\gamma$ as function of $S_{v}(0)$ is model-dependent and varies from saturation (14) to $\sqrt{S_{v}(0)}$-growth (8).

In summary, we developed non-perturbative theory of qubit dephasing within two models of Gaussian and non-Gaussian low-frequency noise and performed Monte
Carlo simulations of qubit dynamics within these models. The theory agrees well with simulations and shows that the decoherence strength is controlled by the noise spectral density at zero frequency. It allows for generalizations in several experimentally-relevant directions and should be useful for analysis of observed shapes of quantum qubit oscillations.

This work was supported in part by ARDA and DOD under the DURINT grant \# F49620-01-1-0439 and by the NSF under grant \# 0325551. The authors would like to thank T. Duty, K. Likharev, J. Lukens, Yu. Makhlin, Y. Nakamura, Yu. Pashkin, and A. Schnirman for useful discussions.

[1] Y. Nakamura, Yu.A. Pashkin, and J.S. Tsai, Nature 398, 786 (1999).

[2] J.R. Friedman, V. Patel, W. Chen, S.K. Tolpygo, and J.E. Lukens, Nature 406, 43 (2000).

[3] C.H. van der Wal, A.C.J. ter Haar, F.K. Wilhelm, R.N. Schouten, C. Harmans, T.P. Orlando, S. Lloyd, and J.E. Mooij, Science 290, 773 (2000); I. Chiorescu, Y. Nakamura, C.J.P.M. Harmans, and J.E. Mooij, Science 299, 1869 (2003).

[4] D. Vion, A. Aassime, A. Cottet, P. Joyez, H. Pothier, C. Urbina, D. Esteve, and M.H. Devoret, Science 296, 886 (2002).

[5] J.M. Martinis, S. Nam, J. Aumentado, and C. Urbina, Phys. Rev. Lett. 89, 117901 (2002).

[6] E. Il'ichev, N. Oukhanski, A. Izmalkov, Th. Wagner, M. Grajcar, H.-G. Meyer, A. Yu. Smirnov, A.M. van den Brink, M.H.S. Amin, and A.M. Zagoskin, Phys. Rev. Lett. 91, 097906 (2003).

[7] T. Duty, D. Gunnarsson, K. Bladh, R.J. Schoelkopf, P. Delsing, cond-mat/0305433

[8] J. Claudon, F. Balestro, F.W.J. Hekking, and O. Buisson, "Coherent oscillations in a current-biased $d c$ SQUID", (2004).

[9] Yu. A. Pashkin, T. Yamamoto, O. Astafiev, Y. Nakamura, D.V. Averin, and J.S. Tsai, Nature 421, 823 (2003).

[10] K. Blum, Density Matrix Theory and Applications, (Plenum, NY, 1991).

[11] Y. Nakamura, Yu.A. Pashkin, T. Yamamoto, and J.S. Tsai, Phys. Rev. Lett. 88, 047901 (2002).

[12] K. Rabenstein, and D. V. Averin, Turk. J. Phys. 27, 313 (2003); cond-mat/0310193

[13] E. Paladino, L. Faoro, G. Falci, and R. Fazio, Phys. Rev. Lett. 88, 228304 (2002); E. Paladino, L. Faoro, G. Falci, Adv. Sol. State Phys. 43, 747 (2003); T. Itakura and Y. Tokura, Phys. Rev. B 67,195320 (2003); Y.M. Galperin, B.L. Altshuler, D.V. Shantsev, cond-mat/0312490

[14] N.V. Prokof'ev and P.C.E. Stamp, Rep. Prog. Phys. 63, 669 (2000).

[15] Yu. Makhlin and A. Shnirman, JETP Lett. 78, 497 (2003); cond-mat/0308297

[16] A.I. Burshtein, JETP 21567 (1965). 